\title{
Monitoring Enzyme Reaction and Screening Enzyme Inhibitor Based on MALDI-TOF-MS Platform with a Matrix of Oxidized Carbon Nanotubes
}

\author{
Ligang $\mathrm{Hu}^{*}$ and Guibin Jiang \\ Research Center for Eco-Environmental Sciences, Chinese Academy of Sciences, Beijing, China \\ Songyun $\mathrm{Xu}$, Chensong Pan, and Hanfa Zou \\ Dalian Institute of Chemical Physics, Chinese Academy of Sciences, Dalian, China
}

\begin{abstract}
A matrix assisted laser desorption/ionization time-of-flight mass spectrometry platform for quantitatively monitoring enzyme activity and screening enzyme inhibitors has been demonstrated. The described method employs a new matrix of oxidized carbon nanotubes. Compared with the traditional fluorescence approach, this label-free method has the advantage of directly identifying the substrates and products in enzymatic reactions. Moreover, the method could be conveniently carried out with any commercial mass spectrometer without modification. We quantitatively monitored the acetylcholinesterase activity and screened acetylcholinesterase inhibitors with a detection rate of about $3.3 \mathrm{~s}$ per sample. (J Am Soc Mass Spectrom 2006, 17, 1616-1619) (c) 2006 American Society for Mass Spectrometry
\end{abstract}

$\mathrm{F}$ luorescence plate reader is the dominant tool for screening enzyme inhibitor [1-6]. They are simple, sensitive, and can be employed in an automated mode and high-throughout format. Nevertheless, the incorporation of fluorescence moiety into the substrate molecules could sometimes affect the enzyme activity. The false positives or false negatives caused by unwanted fluorophore interactions and fluorescencequenching sometimes may occur. As a rapid, sensitive, and direct approach to characterize the analyte qualitatively and quantitatively, mass spectrometry has been utilized to monitor enzyme-catalyzed reactions and to screen enzyme inhibitors. It can effectively complement the traditional fluorescence methods [6-8]. However the matrix effect and poor quantitative capability of MALDI-TOF-MS, and low throughput of LC-MS have limited their further applications.

The platform described here is based on MALDITOF-MS with a matrix of oxidized carbon nanotubes. Compared with traditional organic matrices, MALDITOF-MS with a matrix of oxidized carbon nanotubes succeeds in eliminating the background in low mass

Published online August 14, 2006

Address reprint requests to Dr. G. Jiang, State Key Laboratory of Environmental Chemistry and Ecotoxicology, Research Center for Eco-Environmental Sciences, Chinese Academy of Sciences, 18 Shuangqing Road, Beijing, China 100085. E-mail: gbjiang@rcees.ac.cn

* Also at the Dalian Institute of Chemical Physics, Chinese Academy of Sciences, Dalian, China. area, and provides results with good reproducibility and accuracy for quantitative analysis [9-11]. During the desorption/ionization process, carbon nanotubes serve as both the energy receptacle for laser radiation and the energy transporter for desorption/ionization of analytes. The way to use the matrix of oxidized carbon nanotubes is similar to the thin-layer method in conventional MALDI-TOF-MS. The matrix solution of carbon nanotubes was deposited on the sample target to form a thin-layer of carbon nanotubes, and then the analyte solution was added. The employed commercial MTP MALDI target, which is in the microtiter plate format, is an ideal interface that allows transferring samples onto the target with laboratory robots. Therefore, the high-throughput and automated analysis can be easily achieved on commercial instruments. Using this method, acetylcholinesterase-catalyzed reactions were analyzed and potential inhibitors of acetylcholinesterase were screened. The detection rate could be up to 3.3 s per sample.

\section{Experimental \\ Instrumentation}

The mass spectrometry measurements were performed with an Autoflex (Bruker Daltonics, Bremen, Germany) MALDI-TOF-MS. The sample was deposited onto a ground-steel sample target or an AnchorChip (Bruker 
Daltonics) target with 384 spots, and irradiated with a nitrogen laser at $337 \mathrm{~nm}$ with a neutral density filter. The measurements were performed in either positive or negative ionization mode.

All analyses were performed in the automated mode, which was designed to search for an analyte signal from each sample spot by monitoring the signal-to-noise ratio and peak resolution as a function of laser position. The data would be valid when the signal-to-noise ratio and the resolution of evaluated peak exceed the threshold, which is typically set at 3 and 100, respectively. The laser intensity was initially adjusted to and kept at an appropriate level that would be of great benefit to quantitative analysis.

\section{Chemicals and Materials}

All reagents were analytical-grade unless mentioned otherwise. Acetylcholinesterase (type VI-S, from an Electrophorus Electrics) and tacrine were purchased from Sigma (St. Louis. MO). Multiwalled carbon nanotubes was kindly offered by Professor Wei F. (Tsinghua University, Beijing, China). Methanol, acetone, and acetonitrile were purchased from Kemiou Chemicals (Tianjin, China). All of the candidates for screening enzyme inhibitors were purchased from J \& K Chemicals (Beijing, China). The water used in the experiments was prepared by a Milli-Q system (Millipore, Milford, MA).

\section{Preparation of the Matrix of Carbon Nanotubes}

Because of its poor solubility, the raw carbon nanotube is difficult to be deposited onto the target to form a homogeneous layer. Therefore, it should be treated with an oxidization procedure before using it as the matrix. The method of preparation of the oxidized carbon nanotubes has been described in our previous papers $[10,11]$.

\section{Preparation of Analyte Solution}

The layers of oxidized carbon nanotubes are hydrophilic, and pure organic solvents will spread across on it. Therefore, analytes for direct detection were normally dissolved in water or water/methanol (1:1). Analytes for the screening of enzyme inhibitors were normally dissolved in methanol or water with the concentration of $1.0 \mathrm{mM}$.

\section{Acetylcholinesterase Assay}

Typically, the reaction mixture of acetylcholinesterase $(0.225 \mu \mathrm{M})$, candidate chemicals $(20 \mu \mathrm{M})$ and acetylcholine $(10 \mu \mathrm{M})$ prepared in ammonium bicarbonate buffer $(10 \mathrm{mM}, \mathrm{pH}=8.0)$ to a final volume of $100 \mu \mathrm{L}$ was incubated for $30 \mathrm{~min}$ at $37^{\circ} \mathrm{C}$. Fifty $\mu \mathrm{L}$ acetonitrile was then added to the mixture to terminate the reaction. Finally, aliquot of $1 \mu \mathrm{L}$ was deposited onto oxidized carbon nanotubes layer for further MS analysis.

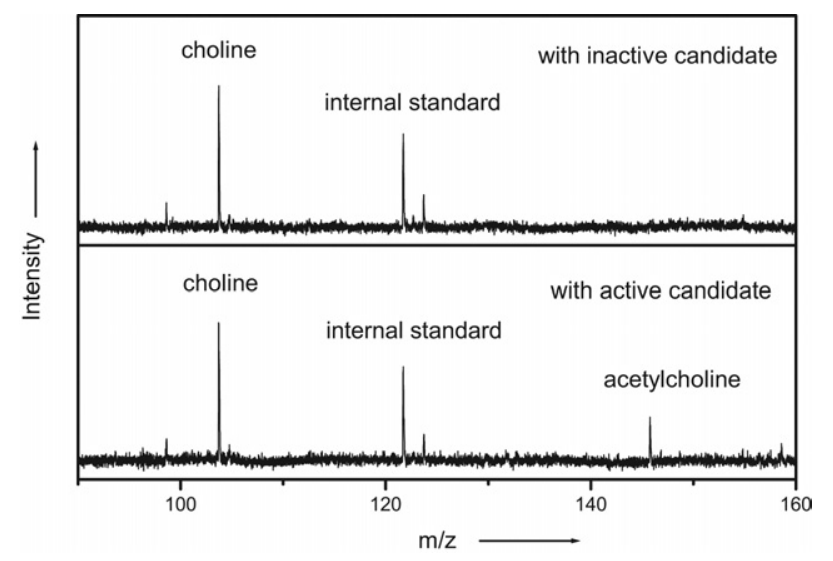

Figure 1. Typical mass spectra for enzymatic hydrolysis reaction of acetylcholine to choline under the existence of acetylcholinesterase with active or inactive inhibitor. Under optimized conditions, the acetylcholinesterase-catalyzed conversion of acetylcholine to choline would proceed completely with the inactive inhibitor or without the inhibitor, but incompletely with an active inhibitor.

\section{Results and Discussion}

\section{Quantitative Analysis in the Automated Mode}

Quantitative analysis of low mass compounds by MALDI-TOF-MS with a matrix of carbon nanotubes has been described in detail in our previous papers [10,11]. Here, quantitatively monitoring of enzyme activity in the automated mode was demonstrated. As a structural analog, chlormequat was chosen as the internal standard for quantitative analysis of acetylcholine and choline. Relative standard deviation of six spots of 10 $\mu \mathrm{M}$ acetylcholine (with the internal standard of $5 \mu \mathrm{M}$ ) in the automated mode was less than $10 \%$. There was also no significant difference between the results obtained from automated mode and manual mode. Thus, all the latter experiments were performed in the automated mode unless mentioned otherwise. Although the linear relationship of the calibration curve obtained on the AnchorChip target was slightly better than that on the ground-steel target, the ground-steel target was employed in our experiments because the diameter (between 200 to $800 \mu \mathrm{m}$ ) of the AnchorChip target is too small to accurately deposit the sample solution by hand. A good linear relationship of calibration curve for acetylcholine was obtained $\left(R^{2}>0.99\right)$, which indicates the accuracy of quantitative analysis in the automated mode.

Using the method of MALDI-TOF-MS with the matrix of carbon nanotubes, the reaction of hydrolyzing acetylcholine to choline catalyzed by acetylcholinesterase in vitro was quantitatively monitored. As shown in Figure 1, the hydrolyzing reaction is observed through the appearance of choline and disappearance of acetylcholine. Acetylcholine was selected as the target compound to evaluate the reaction quantitatively. Furthermore, the limit of detection could reach $1.0 \mu \mathrm{M}$ (S/N > 3). Figure 2 shows the kinetic plot of the hydrolysis of 


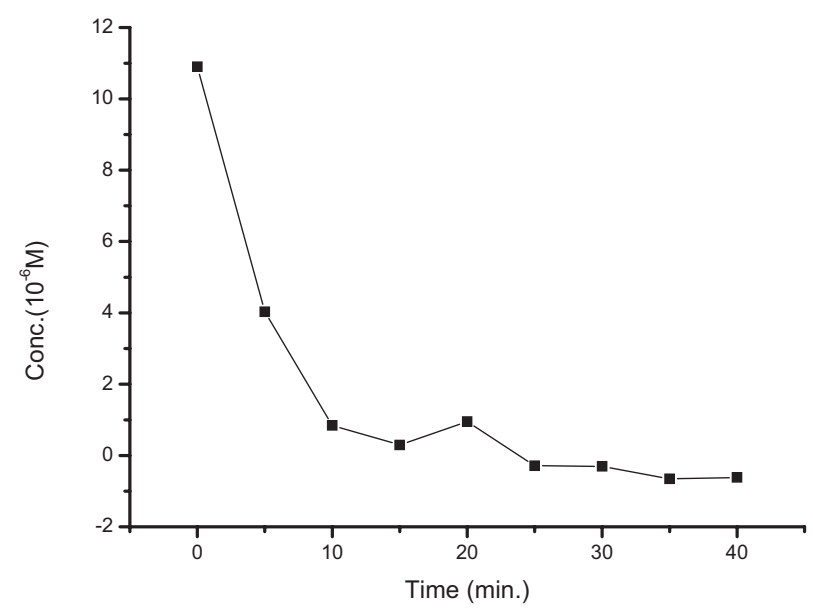

Figure 2. Kinetic plot of acetylcholinesterase-catalyzed hydrolysis of acetylcholine to choline.

acetylcholine catalyzed by acetylcholinesterase. The reaction rate is the fastest at the beginning and then slows down, and the reaction could reach completion within $30 \mathrm{~min}$. When performing kinetic analysis, the average reaction rate within the first 2 min was considered as the initial rate of the reaction, which was obtained in different concentration of substrate ranged from 10 to $80 \mu \mathrm{M}$. The linear regression equation for the Linewever-Burk is: $\mathrm{Y}=125.22 \mathrm{X}+0.5334\left(\mathrm{R}^{2}=0.9923\right)$, and the calculated kinetic parameters are $\mathrm{V}_{\max }=1.87 \mu \mathrm{M} / \mathrm{S}$ and $\mathrm{K}_{\mathrm{m}}=0.23 \mathrm{mM}$.

In our experiments, the reaction mixture of acetylcholinesterase, candidate chemicals, and acetylcholine was incubated for $30 \mathrm{~min}$ at $37^{\circ} \mathrm{C}$, then a terminating agent was added. To terminate the enzyme reaction quickly, a different agent and a different volume of terminating agent were investigated. Acetonitrile and acetone were found to be the appropriate terminating agents that not only can terminate the reaction rapidly but also are compatible with the final MS detection. Acetonitrile was chosen as the terminating agent, and a volume of $50 \mu \mathrm{L}$ was found to be appropriate. Compared with the conventional method, the analyte solution tends to crystallize more homogeneously on the layer of oxidized carbon nanotubes. This facilitated a rapid finding of the sweet spot. Consequently, it speeded up the measurement by locating the laser easily in the automated mode.

The screening of potential acetylcholinesterase inhibitors was performed by monitoring the acetylcholinesterase activity in the presence of candidate compounds. Under optimized condition, acetylcholine is completely hydrolyzed to choline within $30 \mathrm{~min}$. The remaining acetylcholine indicates the inhibition potency of the compound. As shown in Figure 3, acetylcholine could not be hydrolyzed completely to choline within $30 \mathrm{~min}$ because of the inhibition by arsenocholine, malathion, methamidophos, parathion, phorate, and paraquat on acetylcholinesterase. The inhibition rate was determined by the ratio of peak intensity of acetylcholine to

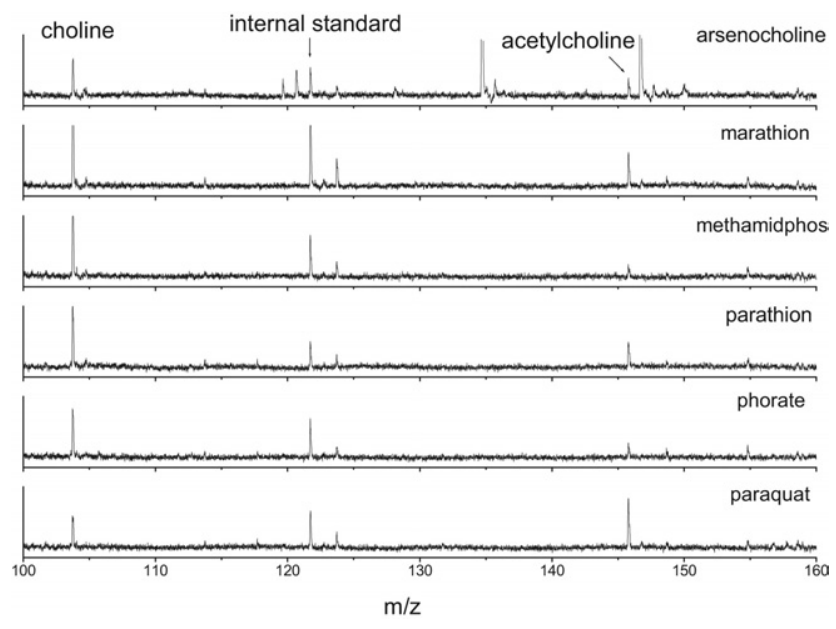

Figure 3. MS spectra for screening of inhibitors.

chlormequat. In our experiment, chlormequat was used not only as an internal standard for quantitative analysis but also as a standard to evaluate spectrum quality and control the data accumulation in automated mode. Only the data with peak of chlormequat exceeding the threshold can be accepted.

Among the candidate compounds, organophosphors (malathion, parathion, methamidophos, phorate), paraquat, and arsenocholine were identified as acetylcholinesterase inhibitors through the high ratios of the peak intensity of acetylcholine to chlormequat. The identified acetylcholinesterase inhibitors are all previously known except for paraquat and arsenocholine which are identified for the first time in this study. The detailed results are listed in Table 1. The throughput of the screening assay was $\sim 3.3 \mathrm{~s}$ per sample, and $190 \mathrm{~s}$ for a set of 50 samples with a $20 \mathrm{~Hz}$ laser frequency. In our experi-

Table 1. Screening results of acetylcholinesterase inhibitors among 40 environmentally relevant chemicals. Under the described reaction condition, six candidates would significantly inhibit the acetylcholinesterase-catalyzed conversion acetylcholine to choline

\begin{tabular}{lc}
\hline Candidate compound & $\begin{array}{c}\text { Conversion rate of } \\
\text { acetylcholine (\%) }\end{array}$ \\
\hline \hline Arsenocholine & 75 \\
Malathion & 61 \\
Methamidophos & 63 \\
Parathion & 37 \\
Phorate & 60 \\
Paraquat tetrahydrate & 24 \\
34 Types compounds* & 100 \\
\hline
\end{tabular}

The 34 type of compounds listed as below: 3,4-xylidine, 4-chloroaniline, 2,4-dichloroaniline, 2,4,6- trichloroaniline, 2-nitroso-1-naphthol, 2-naphthol-3,6-disulfonic acid disodium salt, phenyltin trichloride, diphenyltin dichloride, triphenyltin chloride, butyl trichloro tin, dibutyl dichloro tin, tributylchlorostannane, dibutyltin dilaurate, trichloromethylstannane, dimethyltin dichloride, trimethyltin chloride, anthracene, phenanthrene, 9h-fluorene, diphenylolpropane, nonylphenol, octyl phenol, cacodylic acid, methane arsonate, arsenous acid, arsenic acid, arsenobetaine, 2-hydroxy atrazine, desisopropylatrazine, atrazine-desisopropyl-2-hydroxy, desethylatrazine, methyl mercury chloride, diquat dibromide monohydrate, chlormequat chloride. 
ments, the manual mode of sampling and the minimum volume of the pipette restrict the final volume $(>100$ $\mu \mathrm{L})$ of the enzymatic reaction. If a smaller volumedispensing system were employed, the final volume of the enzymatic reaction could be reduced to $10 \mu \mathrm{L}$ or even less to match the high sensitivity and small sampling volume of MALDI-TOF-MS.

\section{Conclusions}

A MALDI-TOF-MS platform for screening enzyme inhibitors and quantitatively monitoring enzyme activity has been demonstrated. The method is fast, sensitive, accurate, and simple. Compared with the traditional fluorescence approach, this label-free method offers the advantage of directly identifying the substrates and products of enzymatic reactions. Furthermore, highthroughput screening of enzyme inhibitors or quantitatively measuring of low mass compounds could be conveniently carried out with commercial MALDITOF-MS instruments.

\section{Acknowledgments}

This work was jointly supported by National Basic Research Program of China (2003CB415001), National Natural Science Foundation of China (20327002), and the China State Key Basic Research Grants (2003CB716002).

\section{References}

1. Funeriu, D. P.; Eppinger, J.; Denizot, L.; Miyake, M.; Miyake, J. Enzyme Family-Specific and Activity-Based Screening of Chemical Libraries Using Enzyme Microarrays. Nat. Biotechnol. 2005, 23, 622-627.

2. Myszka, D. G. Analysis of Small-Molecule Interactions Using Biacore S51 Technology. Anal. Biochem. 2004, 329, 316-323.

3. Roda, A.; Manetta, A. C.; Portanti, O.; Mirasoli, M.; Guardigli, M.; Pasini, P.; Lelli, R. A Rapid and Sensitive 384-Well Microtiter Format Chemiluminescent Enzyme Immunoassay for 19-Nortestosterone. $\mathrm{Lu}$ minescence 2003, 18, 72-78.

4. Bobzin, S. C.; Yang, S.; Kasten, T. P. LC-NMR: A New Tool to Expedite the Dereplication and Identification of Natural Products. J. Ind. Microbiol. Biot. 2000, 25, 342-345.

5. Cheng, T. J.; Brik, A.; Wong, C. H.; Kan, C. C. Model System for High-Throughput Screening of Novel Human Immunodeficiency Virus Protease Inhibitors in Escherichia coli. Antimicrob. Agents Chemother. 2004, 48, 2437-2447.

6. Shen, Z.; Go, E. P.; Gamez, A.; Apon, J. V.; Fokin, V.; Greig, M.; Ventura, M.; Crowell, J. E.; Blixt, O.; Paulson, J. C.; Stevens, R. C.; Finn, M. G.; Siuzdak, G. A Mass Spectrometry Plate Reader: Monitoring Enzyme Activity and Inhibition with a Desorption/Ionization on Silicon (DIOS) Platform. Chem. Biochem. 2004, 5, 921-927.

7. Bothner, B.; Chavez, R.; Wei, J.; Strupp, C.; Phung, Q.; Schneemann, A.; Siuzdak, G. Monitoring Enzyme Catalysis with Mass Spectrometry. J. Biochem. 2000, 275, 13455-13459.

8. Schluter, H.; Jankowski, J.; Rykl, J.; Thiemann, J.; Belgardt, S.; Zidek, W.; Wittmann, B.; Pohl, T. Detection of Protease Activities with the Mass Spectrometry Assisted Enzyme Screening (MES) System. Anal. Bioanal. Chem. 2003, 377, 1102-1107.

9. Xu, S. Y.; Li, Y. F.; Zou, H. F.; Qiu, J. S.; Guo, Z.; Guo, B. C. Carbon Nanotubes as Assisted Matrix for Laser Desorption/Ionization Timeof-Flight Mass Spectrometry. Anal. Chem. 2003, 75, 6191-6195.

10. Pan, C.; Xu, S.; Hu, L.; Su, X.; Ou, J.; Zou, H.; Guo, Z.; Zhang, Y.; Guo, B. Using Oxidized Carbon Nanotubes as Matrix for Analysis of Small Molecules by MALDI-TOF-MS. J. Am. Soc. Mass Spectrom. 2005, 16, 883-892.

11. Hu, L.; Xu, S.; Pan, C.; Yuan, C.; Zou, H.; Jiang, G. Matrix-Assisted Laser Desorption/Ionization Time-of-Flight Mass Spectrometry with a Matrix of Carbon Nanotubes for the Analysis of Low-mass Compounds in Environmental Samples. Environ. Sci. Technol. 2005, 39, 8442-8447. 